\title{
La agenda pendiente de la educación inicial y preescolar en México: sexualidad integral
}

\author{
FERNANDO SALINAS-QUIROZ \\ ADRIANA LEONA ROSALES MENDOZA** \\ Universidad Pedagógica Nacional - México
}

Recibido el 02-07-2015; primera evaluación el 16-11-2015; segunda evaluación el 17-02-2016; aceptado el 17-02-2016

\section{Resumen}

En este ensayo argumentamos sobre la necesidad de incorporar educación integral de la sexualidad (EIS) en educación inicial (EI) y educación preescolar (EP) en México. Efectuamos un análisis de contenidos en documentos normativos y programas dirigidos a población de cero a seis años a nivel local y latinoamericano. Como resultados, encontramos que hace falta instrumentar acciones de educación sexual en los centros de EI y EP mexicanos. Nuestra propuesta se basa en algunas aportaciones del enfoque sociocultural construccionista de la sexualidad, y en las perspectivas de género y derechos humanos. Concluimos que las relaciones de

\footnotetext{
* Licenciado en Psicología por la Universidad Iberoamericana (UIA), diplomado Internacional de la Parentalidad y magíster en Psicoterapia Psicoanalítica de Niños y Adolescentes por la Asociación Psicoanalítica Mexicana (APM). Doctor en Psicología, Universidad Nacional Autónoma de México (UNAM). Profesor-investigador titular A de la Universidad Pedagógica Nacional (UPN). Integrante del Sistema Nacional de Investigadores (SNI) nivel C y del Programa para el Desarrollo Profesional Docente (PRODEP). Tercer lugar del Premio Nacional Silvia Macotela 2013-2014. Docente en la Licenciatura en Psicología Educativa y en la Especialización en Educación Integral de la Sexualidad. Contacto: fsalinas@upn.mx

** Licenciada en Sociología por la Universidad Autónoma Metropolitana (UAM), Xochimilco. Especialista en Estudios de la Mujer por El Colegio de México. Maestra y doctora en Ciencias Antropológicas por la UAM, Iztapalapa, en donde obtuvo la medalla al Mérito Académico «Incalli Ixcahuicopa» en la maestría y en el doctorado. Es profesora-investigadora titular C de la Universidad Pedagógica Nacional (UPN). Integrante del Sistema Nacional de Investigadores (SNI) nivel 2 y del Programa para el Desarrollo Profesional Docente (PRODEP). Contacto: leonarosales@gmail.com
} 
base segura resultan cruciales para la construcción de vínculos de apego nińo-educador, y que dichos vínculos potencian el desarrollo humano en todas sus esferas, particularmente el desarrollo sexual.

Palabras clave: educación sexual, perfeccionamiento docente, prevención, abuso sexual, educación inicial, educación preescolar.

\section{Comprehensive Sexuality Education: A pending matter in Early Childhood Education in Mexico}

\section{Abstract}

This paper stresses the need to incorporate Comprehensive Sexuality Education (CSE) during the Early Childhood and Preschool school stages in Mexico. A detailed analysis of the content of strategies, courses of action and policies for early education was made for both, the local and Latin-American level. Findings indicate that there remains a need for CSE for 0 to 6-year-old Mexican children. The inclusion of a human rights and a sociocultural perspective of gendered sexuality is strongly recommended. Early life-course interactions between children and their caregivers have the potential to influence developmental outcomes in an enduring manner because they become internalized in the form of generalized mental representations. CSE encompasses the history of care where consistent and coherent secure base support is needed.

Keywords: Sexual education, professional development, prevention, sexual abuse, early childhood education.

\section{A agenda pendente da Educaçáo Inicial e Pré-escolar no México: Sexualidade Integral}

\section{RESUMO}

No presente trabalho iremos abordar a necessidade de incorporar a Educaçáo Integral da Sexualidade (EIS) em Educação Inicial e Pré-escolar no México. Realizamos uma análise dos conteúdos em documentos normativos e programas dirigidos as crianças entre o zero e os seis anos a nível local e latino-americano. Os resultados indicam que faz falta empreender acçóes de educação sexual nos Centros de Educação Infantil e Pré-escolar mexicanos. A nossa proposta tem por base as contribuiçóes das abordagens socioculturais da sexualidade, a perspectiva de gênero, e os direitos da infância. Concluímos que as relaçôes de base segura são fundamentais para a construçáo de vínculos afetivos criança-educador e que os vínculos incentivam o desenvolvimento humano integral, particularmente o desenvolvimento sexual.

Palavras-chave: educação sexual, formação continua dos professores, prevenção, abuso sexual, educação inicial, educação pré-escolar. 


\section{INTRODUCCIÓN}

En este ensayo de tipo argumentativo abordamos la problemática de la inclusión de la educación integral de la sexualidad (EIS), con la finalidad de mostrar la necesidad de crear estrategias para lograr su incorporación en educación inicial (EI) y educación preescolar (EP). Lo anterior constituye un reto que los diseñadores de políticas públicas educativas mexicanas deben tener en cuenta, en función de aportar soluciones a situaciones que enfrentan niñas y niños en su vida cotidiana, como la violencia sexual y la socialización basada en estereotipos de género que reproducen la desigualdad entre hombres y mujeres desde el inicio de la infancia. Consideramos que hay que elaborar e incluir contenidos sobre EIS, con perspectivas de género y derechos humanos en los programas de EI y EP, pues si bien esto se ha logrado en primaria y secundaria, se ha desestimado su relevancia para las nińas y los nińos de cero a seis ańos de edad. Con ellos, se requiere trabajar sobre el conocimiento del cuerpo, resaltando su derecho a la integridad física y emocional. Esto es trascendental, ya que la problemática del abuso sexual es notable. Sugerimos que dado que en EI y EP se establecen relaciones con personas distintas a las del entorno familiar, los educadores pueden convertirse en figuras de base segura y apoyar el desarrollo socioemocional de los nińos y las niñas, ya que la confianza en los otros y en uno mismo se relaciona con la EIS y resulta en la prevención del abuso sexual infantil.

En México, la EI se enfoca en infantes de cero a tres años, y la EP en niñas y niños de tres ańos cumplidos a seis. En otros países de la región los rangos de edad son distintos. Por ejemplo, en Argentina la EI incluye a niñas y niños de 45 días a cinco años de edad, quienes acuden al jardín de infantes. En Nicaragua comprende de cero a cinco ańos. En Uruguay de tres a cinco. En Chile, infantes de cero a tres años acuden a educación parvularia, y a niveles de transición entre los cuatro y los seis. En Colombia, la EI se cursa de cero a seis en centros de desarrollo infantil. En Guatemala, para el mismo rango de edad, hay un sistema de educación inicial y preprimaria, y en Cuba, los niños de cero a seis se inscriben en educación preescolar (Camacho, s.f.).

En 2010 la población infantil mexicana de cero a seis años era de 12693101 personas: 6448940 varones y 6244161 mujeres, lo que representaba el 11,3\% de la población total del país. De cero a tres años había 8342856 infantes (Inegi, 2010). Sobre la asistencia a EI de la población de cero a tres años, la información es escasa; pero se estima que estaban inscritos 1005954 infantes. De los tres a los seis ańos se consigna que asistían a EP 5483 878, de los cuales 2769593 eran varones y 2714 285, mujeres (Inegi, 2010). 
La EI no es obligatoria, y la EP adquirió ese carácter en 2002, hecho que posicionó a México como puntero a nivel latinoamericano. Para EI, en 2012 —durante el último año de gobierno del presidente Felipe Calderón- la ONU premió a la administración por el Programa de Guarderías y Estancias Infantiles para apoyar a madres trabajadoras 2007-2012 (Sedesol, 2012), de manera que todo parecía miel sobre hojuelas; sin embargo, desde que se dio a conocer la noticia, un sector de la sociedad civil cuestionó dicha celebración con base en el incendio de la guardería $\mathrm{ABC}$, ocurrido en el estado de Sonora en el ańo 2009, en donde murieron 49 infantes; sobre todo, porque en todo el país estos espacios de atención infantil habían atravesado por un proceso de subrogación a particulares por parte del Estado, con consecuencias como la carencia de planes de seguridad y bienestar para los infantes. De esta manera, se hizo visible que el cuidado hacia la primera infancia se veía más como un negocio lucrativo que como un espacio educativo. Inclusive, la denominación como "guarderías» y/o "estancias» infantiles evidencia una visión asistencialista, centrada en el adulto, que conceptualiza el servicio como una prestación laboral de la madre y/o el padre, en donde "guardan» a sus hijos o ahí se "están» (Salinas-Quiroz, 2014). Asimismo, llamar al nivel educativo que abarca de los cuatro a los seis ańos "preescolar» le resta relevancia y lo posiciona como un servicio preparatorio y de segunda. Lo anterior nos lleva a cuestionar la terminología empleada para hacer referencia a la EI y EP, dado que esos recintos tienen más la finalidad de acoger a las niñas y los niños que de insertarlos en un proceso educativo. En ese sentido, consideramos que resultaría más adecuado nombrarlos como "centros de educación inicial», ya que las y los infantes tienen el derecho a recibir, además de cuidado, atención educativa de calidad, independientemente de los derechos laborales de sus madres y padres (Salinas-Quiroz, 2014), con un interés público superior que los beneficie en términos de su desarrollo y derechos humanos.

En este contexto, y en un intento por contar con un "piso común» entre las distintas instituciones que ofrecen cuidado y atención educativa para EI en México, la Subsecretaría de Educación Básica de la Secretaría de Educación Pública lanzó en 2013 el Modelo de Atención con Enfoque Integral para la Educación Inicial (SEP, 2013). Pese a que se trata de un ejercicio valioso, en ningún apartado del documento se hace mención explícita a la EIS, aunque puede leerse entre líneas que tendría cabida en uno de sus fundamentos: el enfoque de derechos. Dicho modelo se refiere solo a la igualdad adultos-nińos, y no considera el género (lo que podría representar una base para abordar la EIS). Otra problemática que atraviesa la inclusión de contenidos de EIS en los centros de EI, es que, al menos seis instituciones distintas ofrecen servicios 
en este nivel educativo, lo que no propicia que los infantes reciban servicios de atención educativa de la misma calidad y bajo estándares homologables, ya que cada una de las instituciones tiene regulaciones y programas internos.

En el ámbito de la educación sexual, una de las metas para 2015 de la Declaración Ministerial: Prevenir con Educación (2008) era reducir en un $75 \%$ la brecha en el número de escuelas que no hubiesen institucionalizado la EIS. De acuerdo con un diagnóstico efectuado por el Programa Regional para América Latina y el Caribe del Fondo de Población de las Naciones Unidas, Argentina, Uruguay, Colombia, República Dominicana, Cuba, Perú, Nicaragua, Paraguay y México han incorporado la EIS de manera obligatoria en distintos niveles educativos; pero en EI y EP solo lo han hecho Argentina, Uruguay, Colombia, Cuba, Guatemala, Nicaragua y Chile (Camacho, s.f., pp. 14-15). En México no se consideró prioritario ofrecer EIS a la población de cero a seis años, a pesar de que el Programa de Educación Preescolar (PEP) había logrado la incorporación de la perspectiva de género en 2011. En comparación con México, Argentina cuenta con un programa más definido para el trabajo sobre EIS en EI, el cual:

[...] favorece e incluye el conocimiento y cuidado del propio cuerpo y el de las y los demás; la valoración de las emociones y expresiones; la información básica sobre la dimensión biológica de la sexualidad [...] el fomento de valores y actitudes relacionados con el amor, la solidaridad, el respeto por la vida y la integridad de las personas [...] y el ejercicio de los derechos relacionados con la sexualidad (Marina, 2010, p. 12).

En México no se cuenta con información suficiente sobre violencia sexual para la población de cero a seis años. En los datos aportados por la Agenda Sectorial para la Educación Integral de la Sexualidad (Secretaría de Educación Pública [SEP], 2015) se señala que, de acuerdo con el Fondo de las Naciones Unidas para la Infancia (Unicef), entre las causas de la violencia contra niños, niñas y adolescentes en México se encuentra la percepción social de que estos son propiedad de los adultos. Asimismo, se consigna de acuerdo con datos del Sistema Nacional para el Desarrollo Integral de la Familia (DIF), en 2010 se atendieron 32068 reportes por maltrato infantil, en 17800 de los cuales se comprobó que había ocurrido, de estos el 50,2\% eran niñas (SEP, 2015). Algunos otros datos disponibles se centran en niñas y niños de 6 a 15 años de edad.

El objetivo de este ensayo es argumentar sobre la necesidad de desarrollar estrategias de EIS en EI y EP que se vinculen con los lineamientos establecidos en instrumentos normativos y programas centrados en el desarrollo y 
los derechos humanos de la infancia y la niñez en México, así como sugerir elementos que podrían contener dichas estrategias. En el primer apartado de la sección de resultados, analizamos algunos de estos documentos para mostrar que aunque se menciona que se deberán incorporar temáticas de EIS en instituciones educativas, con contenidos acordes a la edad, esto no ocurre en EI y EP. En el segundo apartado de la sección de resultados reflexionamos sobre la necesidad de visibilizar la sexualidad desde la primera infancia, ya que esta ha sido considerada inexistente por una parte de la literatura especializada, lo que posiblemente ha influido en la concepción de los diseñadores de planes y programas de EI y EP en México. En la sección de discusión y conclusiones, sugerimos que las relaciones de base segura son relevantes para infantes, nińas y nińos de EI y EP, particularmente en el campo de la EIS, y proporcionamos algunas ideas para avanzar en la construcción de una agenda sobre EIS dirigida a dicho sector poblacional.

\section{MÉTodo}

Realizamos un análisis de contenidos sobre EIS en instrumentos normativos y programas que estipulan principios para la atención de infantes, niñas y niños en EI y EP en México, en donde hallamos que existe la necesidad de instrumentar acciones de educación sexual en los centros de atención y escuelas de esos niveles. También percibimos la carencia de una perspectiva teórica capaz de apoyar el diseño de los programas indicativos para EI y EP, como podrían ser los que aquí proponemos: enfoque sociocultural construccionista de la sexualidad; perspectivas de género y derechos humanos, y; teoría del apego. La metodología utilizada es de corte cualitativo, ya que nos basamos en un análisis de contenidos, lo que nos permitió hacer evidente que no basta con mencionar a la EIS en los documentos, sino que hace falta implementar estrategias para su inclusión en planes y programas de EI y EP.

\section{Resultados}

\subsection{Normatividad oficial sobre EIS para infantes y niños en México}

El Plan Nacional de Desarrollo de México 2013-2018 considera promover acciones de desarrollo infantil temprano como una de las líneas de acción dentro de la Meta Nacional México Incluyente, no obstante, esta meta no especifica qué tipo de acciones, ni cómo estas promoverían la inclusión. Además, las llamadas «acciones de desarrollo infantil temprano» se incluyen en la estrategia 
intitulada «Fortalecer el desarrollo de capacidades en los hogares con carencias para contribuir a mejorar su calidad de vida e incrementar su capacidad productiva» (Gobierno de México, 2015), por lo que resulta imposible vincular tales «acciones» con la calidad de la atención educativa, mucho menos con la EIS.

A finales de 2014 se publicó un decreto en el Diario Oficial de la Federación sobre la Ley General de los Derechos de Niñas, Niños y Adolescentes, algunos de sus contenidos resultan pertinentes para el tema que aquí exponemos. El artículo 58, menciona:

La educación, además de lo dispuesto en las disposiciones aplicables, tendrá los siguientes fines [...] Inciso viii: Promover la educación sexual integral conforme a su edad, el desarrollo evolutivo, cognoscitivo y madurez, de las niñas, nińos y adolescentes que les permitan a niñas, niños y adolescentes (sic) ejercer de manera informada y responsable sus derechos consagrados en la Constitución Política de los Estados Unidos Mexicanos, en las leyes y los Tratados Internacionales de los que el Estado mexicano sea parte (Diario Oficial de la Federación [DOF], 2014, p. 23).

Se trata del único artículo donde se hace mención explícita a la EIS, pues, ni el Plan Nacional del Desarrollo, el Modelo de Atención con Enfoque Integral, o el PEP abordan el tema. Debido a que se trata de una ley general, se convierte en una reglamentación con la que tienen que armonizarse otros ordenamientos, por lo que consideramos que constituye un instrumento poderoso para pensar y promover la EIS en EI y EP. Por su parte, la Agenda Sectorial para la Educación Integral en Sexualidad, con énfasis en la prevención del embarazo en adolescentes del ańo 2015 indica que en EP:

[...] se abordan los vínculos afectivos que desarrollan niñas y niños a partir de sus vivencias y roles en la casa y en la escuela; se educa sobre responsabilidades y derechos, sensaciones y sentimientos, y cómo actuar ante determinadas situaciones y/o personas de riesgo (SEP, 2015, p.52).

No obstante, como ya señalamos, para este nivel educativo no existe un programa definido, por lo que es improbable que se lleven a cabo acciones pedagógicas sobre EIS. De acuerdo con la misma Agenda, uno de los sectores a atender son las niñas y niños de tres a cinco años que cursan preescolar, con el objetivo de evitar el abuso sexual (SEP, 2015). Empero, no se indican las estrategias a seguir para eludirlo, ni métodos para prevenirlo, o qué hacer y a dónde acudir en caso de detectar un caso de abuso sexual en la sala. En este sentido, sugerimos que se trata de enunciados de «buena voluntad», 
pues no se han incorporado secuencias didácticas específicas, o un espacio curricular particular para trabajar la EIS en el PEP.

El documento denominado Programa de Estudio 2011. Guía para la Educadora. Educación Básica Preescolar (SEP, 2011) no cuenta con una secuencia de contenidos y actividades (p. 15); posee una modalidad abierta, en donde se da libertad a la educadora para que diseñe actividades con distintos niveles de complejidad, de acuerdo con las posibilidades de cada nińo (p. 13), para lo cual propone enfatizar en el desarrollo de competencias a través de actividades didácticas que impliquen desafíos para las niñas y los niños, con la finalidad de que puedan proponer, distinguir, aplicar, cuestionar, comparar y trabajar en equipo (p. 14). Entre las competencias a desarrollar se encuentran el manejo de emociones, la adquisición de confianza para comunicarse, el reconocimiento de «la diversidad lingüística, cultural, étnica y de género» (p. 18), y que practiquen acciones de preservación de la salud y prevención de riesgos.

En el Programa de Estudio de Preescolar (PEP, 2011) define a la equidad de género (p. 23), pero, no considera a la diversidad sexo-genérica. En dicho programa se habla del cuerpo, pues se supone que los niños de preescolar «entiende[n] algunas interconexiones elementales entre las diferentes partes del cuerpo, tanto internas como externas» (p. 35); se incluye la salud, ya que el nińo «toma decisiones de su vida personal compatibles con su salud» (p. 36), y se valora el desarrollo de actitudes mediante las cuales se «respeta[n] las diferencias raciales, étnicas, de género y sexualidad en la aplicación de la ciencia» (p. 35). Específicamente en el campo formativo de Desarrollo fisico y salud (PEP, 2011) se explica que es relevante que los niños aprendan a tomar decisiones que estén a su alcance para cuidarse a sí mismos (p. 70), y «ayudarles a entender que existen situaciones en las que puede estar en riesgo su integridad personal» (p. 71). Para ello, se estima que «la maestra puede ser una figura importante, de gran influencia, en quien puedan confiar cuando enfrentan situaciones difíciles de maltrato o violencia» (p. 104), por lo que se considera necesario crear espacios en donde conversar sobre las «sensaciones y emociones que experimentan frente a algunos adultos o ante situaciones en las que no se sienten seguros... que les puede provocar miedo o malestar, y a reconocer qué pueden hacer cuando se sienten en peligro» (p. 71). Dentro del campo formativo denominado Desarrollo personal y social, se habla de que las niñas y los nińos han aprendido a identificar y regular emociones con la finalidad de establecer relaciones de empatía (p. 75), y se destacan como "aprendizajes esperados» el reconocimiento de la injusticia y los derechos, así como el sentido de respeto. En el campo Cultura y vida social, se promueve el desarrollo 
de la competencia de «respeto a la diversidad», pero solo en el sentido de las diferencias entre culturas debidas a la pertenencia étnica (p. 67) y no de género o sexual.

En el análisis que realizamos a los contenidos de la Guía para la Educadora y el PEP (2011), se ubica el estudio del cuerpo humano en relación con su aspecto físico, su salud y las capacidades motrices gruesas y finas (p. 69), pero no se vincula con el abuso sexual. Entre los «temas de relevancia social» (p. 133) se hace referencia a la educación sexual, aunque no se incluye en ninguno de los campos formativos, con excepción de un señalamiento sobre abordar las diferencias de género y sexualidad, dentro del cual, de nuevo, no se menciona la diversidad sexo-genérica. Identificamos temas que resultan relevantes y que se pueden vincular con educación sexual, como el manejo de emociones, y el adquirir confianza para comunicarse; no obstante, no hayamos propuestas didácticas o de intervención por parte de la educadora en cuanto a cómo puede apoyar el proceso de interpretación y regulación de las emociones, qué hacer para que niñas y niños reconozcan las situaciones de riesgo; cómo generar confianza y seguridad y de qué forma les puede hablar de una situación peligrosa, como el abuso sexual. Coincidimos con el PEP (2011) en que la figura de la educadora es importante; la confianza que se tenga en ella puede ser de gran influencia para el desarrollo del infante, y se puede constituir en una persona de apoyo en situaciones de maltrato, violencia o abuso sexual, es decir, en una figura de base segura — tema sobre el que profundizamos más adelante-.

\subsection{Sexualidad, educación e infancia}

La educación puede ser definida como un fenómeno social en el cual las personas nos apropiamos de la cultura (Aller, 1994). Dentro del entramado de símbolos, significados y prácticas culturales encontramos a la sexualidad (Rosales, 2012). La sexualidad consiste en un conjunto de relaciones que son específicas histórica y culturalmente (Szasz, 1998) sin distinción de edad e implica expresiones de sensualidad y erotismo, así como caricias no genitales, estando inmersa en relaciones de poder institucionalizadas por el Estado, las relaciones de género y el parentesco (Rosales, 2010). La sexualidad suele circunscribirse al mundo adulto, olvidando que los niños y las niñas tienen un papel activo en la construcción de las relaciones sociales en las que se comprometen y la expresan de igual manera. Como cualquier otra persona, formulan sentidos sobre el mundo que los rodea, por lo que son también productores de cultura. En ese sentido, deseamos subrayar que resulta inadecuado referirnos 
a ellas y ellos como «menores», pues son individuos que cuentan con saberes distintos — no inferiores - a los de los adultos (Reybet, 2009; Salinas-Quiroz, Cambón \& Silva, 2015), por lo que optamos nombrarlos infantes ${ }^{1}$, nińas o niños.

La educación de la sexualidad se ha encaminado típicamente a proporcionar información desde una visión adulto-céntrica que decide en qué etapa del desarrollo se está "preparado" para recibir un cúmulo de datos de manera pasiva. En el medio educativo, la corriente biologicista ha gozado de gran popularidad debido a que considera, desde una epistemología positivista, que únicamente se produce conocimiento mediante el método científico. Esta perspectiva biologicista se ha construido sobre la base de lo que Michel Foucault denominó scientia sexualis, en la cual, el sexo se sitúa en lo «normal» o en lo "patológico». La ciencia de la sexualidad es uno de los aparatos desplegados desde el siglo XVIII para producir «la verdad» sobre el sexo (Foucault, 1993). En esta perspectiva, los cambios hormonales y anatómicos que ocurren en la pubertad son la piedra angular, por lo que gran parte de la educación de la sexualidad se ha enfocado en esto, y ha dejado de lado aproximaciones relacionales/culturales, de manera que en realidad no se logran procesos integrales de enseñanza-aprendizaje sobre la sexualidad, sino que solo se ofrecen visiones parciales. Como consecuencia de lo anterior, educadoras y educadores transmiten roles estereotipados, censuran la curiosidad y el placer; perpetúan tabúes y mitos, y; vinculan a la sexualidad únicamente con lo genital (San Pelayo Ferrer, 2008).

En otros casos, desde concepciones del siglo XVIII, se percibe a las niñas y los nińos como seres asexuales, ubicados en una etapa de inocencia y pureza; período que no debería ser "contaminado» con algo tan «sucio» como la sexualidad (Bobier \& Martin, 2016). Siguiendo esta línea de pensamiento, se plantea que la sexualidad infantil es inoportuna, ya que el proceso «natural» implica que sus manifestaciones inicien en la pubertad, y en ese sentido, no se considera prioritaria la inclusión de contenidos sobre EIS en EI y EP. Desde hace más de un siglo, y contrario a tales concepciones, Sigmund Freud (1905) visibilizó y describió prácticas eróticas desde el nacimiento: propuesta incendiaria y novedosa. Si bien el aporte a la sexualidad infantil desde el psicoanálisis es innegable, la caracterización propuesta por el autor a manera de etapas

1 A lo largo del presente artículo hemos utilizado el término infante por su carácter inclusivo y neutro en cuánto a género se refiere, además de que abarca específicamente a personas de cero a tres ańos de edad. De tres ańos a seis, consideramos más adecuado utilizar «niñas» $\mathrm{y}$ «niños». 
(Tres Ensayos, 1905) ha sido tomada como estandarte para intentar explicar la sexualidad infantil sin cuestionar la confiabilidad y validez de la teoría.

En este sentido, estimamos pertinente que al incluir EIS en programas de EI y EP se incorpore también, de fondo y no solo de forma, la perspectiva de género, pues esto permitirá mirar a infantes, niñas y niños como seres que, de alguna u otra forma, ejercen su sexualidad de manera diferenciada. La formación docente deberá facilitar las intervenciones educativas con enfoque de género, con miras a reconocer la diversidad de formas en que se expresa el género. No es tarea menor, pues agentes educativos y docentes tienden a confundir los conceptos de sexualidad, sexo y género, los cuales son fundamentales para impartir EIS en los diferentes niveles educativos. En términos generales, se ha pensado que nuestra sexualidad nos da identidad, pues «...la cultura moderna ha supuesto que existe una conexión íntima entre el hecho de ser biológicamente macho o hembra...» (Weeks, 1998, p. 17). Por lo tanto, se suele creer que hay que orientar a las niñas y los niños hacia las expresiones «normales» de su feminidad o masculinidad. Esta es una idea de «sentido común» que se toma como verdadera al momento de transmitir conocimientos a los nińos, resultando en una advertencia mutuamente excluyente: o se es femenino o se es masculino, desestimando el tránsito entre distintas identidades sexo-genéricas. Debido a lo anterior, resulta relevante invertir en la formación de educadoras, pues de otra manera se continuarán transmitiendo estereotipos y modelos sobre lo que se considera deberían llegar a ser las mujeres y los hombres. El sexo se refiere a las características sexuales primarias y secundarias, anatómicas y fisiológicas del ser humano, por regla general, se distingue así a mujeres de hombres: se nace con un pene o con una vagina. El género es la construcción sociocultural de la diferencia sexual; por medio de la cual, sobre la evidencia de los genitales, se inscriben mandatos culturales diferenciados para hombres y mujeres (Rosales, 2010).

Los niños y las niñas desarrollan la constancia de género hasta la edad aproximada de tres años (Campbell, 1994; Rocha Sánchez, 2009); «...el reconocimiento de sí mismos como seres sexuados se logra totalmente hacia finales del tercer ańo de vida, cuando comprenden que son ellos los que realizan las acciones, sobre todo, porque son capaces de prescindir del adulto» (Vygotsky, 1991, p. 104), es decir, incorporan su identidad de género antes de reconocer que son iguales o diferentes a otras personas —en términos del sexo biológico-.

La corriente posestructuralista nos invita a salir del pensamiento binario y a reflexionar sobre la infinidad de posibilidades para expresar la sexualidad. Siguiendo esta propuesta es que preferimos hablar de expresiones de género y no 
de identidad de género, ya que aunque actualmente sabemos que la identidad es flexible y cambiable, la utilización del término tiene una tradición que se relaciona con rigidez e inmutabilidad. Para educar en materia de sexualidad es necesario reconocer que mucho de lo que pensamos que somos como mujeres u hombres ha sido establecido mediante un complejo proceso de construcción sociocultural, y que lo que se espera de las niñas y los niños ha sido determinado desde una visión adultocéntrica, heteronormativa y binario excluyente. Quiera o no quiera, cualquier persona que se relacione con otra educa en materia de afectividad y sexualidad. Impartimos educación sexual con lo que decimos y lo que no decimos, con caricias, gestos y abrazos. Las y los adultos educamos y mostramos nuestra propia sexualidad, ya que vivimos en cuerpos sexuados que se expresan en esferas públicas y privadas; educamos la sexualidad infantil con nuestras actitudes y nuestras formas de pensar, sentir y actuar. Es preciso comprender que la educación sexual en la infancia está muy vinculada con el proceso de socialización transmitido por las figuras parentales y los primeros cuidadores.

\section{Discusión y CONCLUSIONES}

\subsection{Importancia de las relaciones de base segura para la EIS en EI y EP}

Los primeros vínculos afectivos se construyen en el entorno cercano, por regla general, el familiar, y de ahí se extienden y amplían a otros espacios. La sexualidad es un proceso que particularmente en los primeros ańos requiere del acompañamiento de figuras sensibles que funcionen como base de seguridad (Salinas-Quiroz, 2014). El ingreso a EI trae consigo nuevas experiencias vinculares con personas ajenas al grupo familiar, de manera que se amplia el horizonte cultural, y puede crearse una red multisistémica de relaciones de base segura (Salinas-Quiroz, 2014; Salinas-Quiroz, Cambón y Silva, 2015). En este sentido, la EIS se convierte en una oportunidad extraordinaria para valorar positivamente las reacciones emocionales que genera contar con cuidadores múltiples y compañeros dentro de un contexto institucional. La disponibilidad de los agentes educativos (cuidadores y/o educadores) es relevante para: la calidad del vínculo que construirá con los infantes, su desarrollo sociocognitivo y éxito escolar, y para educar en sexualidad y género. Como habíamos sugerido, la promoción de relaciones de base segura educador-infante genera beneficios importantes en la EIS, particularmente en la prevención del abuso sexual en niños de cero a seis años. 
Una relación de base segura (también denominada vínculo apego seguro) puede coadyuvar en otros procesos educativos como la participación democrática de los infantes y nińos; la desnaturalización de modelos socioculturales que conciben a los infantes como seres pasivos y vulnerables — valdría la pena preguntarse si no son más bien vulnerados y no vulnerables—, y; los patrones de invisibilización de la sexualidad infantil. Las relaciones de base segura pueden facilitar que se conciba a las personas de cero a seis años como sujetos con derechos, confiriendo prioridad a su desarrollo personal, lo que implica pensar que atraviesan por un proceso de construcción/formación de ciudadanía. Por ello, resulta de vital importancia incorporar también el enfoque de los derechos humanos desde la EIS en EI y EP, ya que la sexualidad es un derecho (Rosales, 2011). Lo anterior concuerda con el artículo 47 de la Ley General de los Derechos de las Niñas, Niños y Adolescentes, que señala que el Estado ha de garantizar que se tomen las medidas necesarias para prevenir, atender y sancionar el abuso sexual, la trata de personas y la explotación sexual infantil (DOF, 2014).

Si bien la inclusión de ejes curriculares transversales en planes y programas de estudio es relevante, pues permite tratar un tema desde diferentes disciplinas y materias, consideramos que la EIS debería impartirse como una asignatura específica. En este caso, será necesario diseñar «lineamientos» para la formación de docentes en los diferentes niveles educativos. En EI y EP el énfasis tendría que ponerse en el desarrollo de conocimientos, actitudes y habilidades sobre perspectiva de género y sexualidad, en donde los agentes educativos pudiesen revisar sus ideas, valores, mitos y prejuicios en torno a la sexualidad adulta e infantil. Asimismo, su formación debería incluir un amplio conocimiento sobre el marco legal de los derechos humanos de la infancia, así como de su aplicación, y la promoción del ejercicio de la ciudadanía (en ciernes) de infantes, niñas y niños. Simultáneamente, se hace necesaria la toma de conciencia en cuanto a la importancia de desarrollar su sensibilidad hacia las necesidades de infantes y niños, pues a través de este mecanismo se promueven las relaciones de base segura, de manera que los infantes/nińos se sentirán con mayor confianza para expresar si han sufrido un abuso sexual, y tendrán la seguridad de que dicha situación puede ser resuelta. La sensibilidad hace referencia a "la habilidad... para estar atenta/o a las señales [del infante]... interpretarlas correctamente, y responder pronta y adecuadamente» (Salinas-Quiroz \& Posada, 2015, p. 1056).

En cuanto a los «aprendizajes esperados» en personas de cero a seis ańos, un programa de EIS debe de ser capaz de lograr que infantes, niñas y niños conozcan el cuerpo humano y sus funciones sexuales básicas; que reconozcan 
las distintas expresiones de género y las potencialidades de las niñas y los niños, con miras a desalentar la reproducción de estereotipos de género; que entiendan sobre sus derechos humanos para propiciar que se les respete como personas, subrayando el cuidado de su cuerpo, no solo en términos de la salud sino, sobre todo, el cuidado socioemocional de sí mismos para evitar el abuso sexual; que desarrollen habilidades para identificar situaciones/individuos poco confiables, y; que aprendan a expresar sus emociones y sentimientos para denunciar abusos de poder y violencia. En este sentido, consideramos relevante el trabajo del educador y su función como figura de base segura.

Cuestionamos la afirmación realizada dentro del campo formativo Desarrollo personal y social del PEP (2011) que indica que las niñas y los niños ya han aprendido a identificar emociones y que se encuentran en proceso de interpretarlas, organizarlas y regularlas con la finalidad de establecer relaciones de empatía, entendimiento y aceptación (p. 77), pues, más bien, consideramos que tanto la regulación emocional, como la construcción de relaciones empáticas, antecede la EP y continúa en años posteriores. Aunado a esto, consideramos que es necesario primero explorar la construcción de los vínculos afectivos de infantes/niños dentro y fuera de los escenarios educativos para, posteriormente, evaluar cómo identifican e interpretan sus emociones. El desarrollo socioemocional está interrelacionado con el desarrollo sexual, pues los vínculos afectivos tienen un nexo con los significados que los adultos atribuyen al ser niño o ser niña; con el uso y abuso del poder; con concepciones acerca de la infancia y la sexualidad infantil, y; con el trato de infantes, niños y nińas como sujetos de derechos.

A lo largo del presente ensayo argumentamos sobre la relevancia de incorporar contenidos sobre educación integral de la sexualidad dirigidos a personas de cero a seis años en los programas de educación inicial y educación preescolar mexicanos, ya que, a través de una revisión bibliográfica, encontramos que en México su inclusión ocurre solamente a partir de educación básica (primaria y secundaria), a diferencia de lo que acontece en Argentina, Uruguay, Colombia, Cuba, Guatemala, Nicaragua y Chile, en donde se han diseñado e implementado programas y contenidos de EIS para EI y EP.

En este sentido, consideramos que ciertos elementos de la propuesta pedagógica argentina son relevantes para la formación de vínculos afectivos, ya que el conocimiento y exploración del contexto; el desarrollo de competencias y habilidades psicosociales; el conocimiento y cuidado del cuerpo, y; el desarrollo de comportamientos de autoprotección (Marina, 2010) se relacionan directamente con el sistema organizado de conductas de base segura que le permiten a los infantes explorar el mundo físico y social con confianza, 
propiciando el aprendizaje (Salinas-Quiroz, 2014). Si la indagación propicia el uso igualitario entre niños y niñas del salón y de los espacios comunes, así como de los juegos y juguetes — desestimando los estereotipos de género-, la EIS estaría conjuntando exitosamente la teoría del apego, la perspectiva de género y el enfoque de derechos humanos.

En México la EIS está ausente en EI y en EP; el PEP (2011) deja abierta la posibilidad de incluirla o no, y la decisión queda en manos de las educadoras que están frente a grupo. Particularmente en el campo de EIS se requiere formar y sensibilizar a docentes para que sean capaces de brindar una educación pertinente a infantes y niños de ambos sexos, de otra manera, transitamos en un terreno incierto, ya que la «transversalidad» de la educación sexual no implica necesariamente que los agentes educativos incorporen el tema en sus acciones pedagógicas.

Por otra parte, hay que tener en cuenta que la sexualidad y el género son dimensiones que atraviesan por estereotipos, prejuicios, mitos, e incluso, miedos personales, lo que impide a educadores y docentes la discusión de estos temas en un grupo. Si no se considera el diseño de programas, contenidos y materiales didácticos de EIS de manera consistente en EI y EP, seremos incapaces de hacer que infantes, niñas y niños se desarrollen en ambientes en los que se sientan seguros; en los que aprendan sobre sí mismos y sus cuerpos; en los que interaccionen con otros individuos de manera armónica; en los que adquieran habilidades para repeler cualquier forma de violencia, sobre todo, el abuso sexual, y; en donde se conciban como sujetos de derechos humanos: como seres que deben ser respetados.

Finalmente, la principal problemática que identificamos en EI es que los agentes educativos no cuentan con la suficiente información y formación en EIS y que pueden limitarse a únicamente "guardar» infantes en «estancias». En EP, el programa correspondiente es ambiguo en la definición de actividades pedagógicas a realizar, en mucho, debido a la libertad que se confiere a la educadora para diseńar e impartir los contenidos. En México, en ambos niveles educativos, la mayor parte del personal está conformado por mujeres, lo que le imprime un sesgo de género a la profesión de educadora, pues refuerza la idea de ellas son más aptas para brindar cuidado y atención a individuos en sus primeros años de vida.

Las áreas de oportunidad en el diseño de programas educativos y en la formación docente en EIS para EI y EP que identificamos son: perspectiva de género; diversidad sexo-genérica; derechos humanos de la infancia; aspectos básicos de la teoría del apego; teoría de las emociones; desarrollo biopsicosocial del infante, y; detección y prevención de la violencia, con énfasis en atención 
del abuso sexual. Adicionalmente, se requiere elaborar y aplicar programas, así como cursos de formación docente de acuerdo con el contexto social — con un enfoque intercultural—, pues las necesidades de infantes, niñas y nińos de cero a seis años son específicas en diferentes zonas urbanas y rurales, así como entre las distintas etnias indígenas existentes en México.

\section{REFERENCIAS BIBLIOGRÁFICAS}

Aller, L. (1994). Pedagogía de la sexualidad humana. Argentina: Galera.

Bobier, L. \& Martin, K.A. (2016). Early childhood sexuality education. En J. Ponzetti \& J. James (Eds.). Evidence-based Approaches to Sexuality Education: A Global Perspective (pp. 201-217). Nueva York: Routledge. En https:// books.google.com.mx/books?hl=es\&lr=\&id=uPyPCgAAQBAJ\&oi= fnd \&pg=PA201\&dq=early+childhood+sexuality+education \&ots=1z_6 MxTvwS\&sig=m54BcGKNfaIiVvMazuARhADFCw\#v=onepage \&q= early\%20childhood\%20sexuality\%20education $\& \mathrm{f}=$ false

Camacho, V. (s.f.). Diagnóstico del Estado del Arte en Educación Integral de la Sexualidad en América Latina y El Caribe (2013-2018). Programa Regional para América Latina y el Caribe, Fondo de Población de las Naciones Unidas. UNFPA, pp. 1-20. En http://www.codajic.org/sites/ www.codajic.org/files/Diagn \%C3\%B3stico\%20del\%20estado\%20 del\%20arte\%20en\%20educaci\%C3\%B3n\%20integral\%20de\%20la $\% 20$ sexualidad\%20en $\% 20 \mathrm{Am} \% \mathrm{C} 3 \%$ A9rica $\% 20$ Latina $\% 20 \mathrm{y} \% 20 \mathrm{el} \% 20 \mathrm{Cari}-$ be\%20Alma\%20Virginia\%20Camacho.pdf

Campbell, A. (1994). Men, woman and agression. Nueva York: Basic Books.

Freud, S. (1905). Tres ensayos de teoría sexual. En S. Freud (Ed.), Obras Completas, VII (pp. 157-2010). Argentina: Paidós.

Foucault, M. (1993). Historia de la sexualidad. La voluntad de saber, 1. México: Siglo XXI.

Gobierno de México. (2015). Plan Nacional de Desarrollo 2013-2019. En http:// pnd.gob.mx/

Gobierno de México-Diario Oficial de la Federación, (DOF) (2014). Ley General de los Derechos de las Niñas, Niños y Adolescentes. México, D.F.

Gobierno de México-Instituto Nacional de Estadística y Geografía (Inegi) (2010). Censo de Población y Vivienda 2010, Tabulados del Cuestionario Básico. Fecha de elaboración 8-02-2011. Población total por Entidad Federativa y edad desplegada según sexo. En www3.inegi.org.mx/.../TabuladosBasicos/ LeerArchivo.aspx?ct...c... 
Gobierno de México-Secretaría de Desarrollo Social (Sedesol). (2012). Programa de Guarderias y Estancias Infantiles para apoyar a madres trabajadoras (20062012). En http://observatoriopoliticasocial.org/sitioAnterior/images/PDF/ programasfed/estancias_infantiles.pdf

Gobierno de México-Secretaría de Educación Pública (SEP) (2015). Subsecretaría de Planeación y Evaluación de Políticas Educativas, Dirección General Adjunta de Igualdad de Género (2015). Agenda Sectorial para la educación integral en sexualidad con énfasis en la prevención del embarazo en adolescentes, Documento de Trabajo de la Segunda Reunión de Trabajo con las Áreas e Instituciones de la SEP, 30 y 31 de julio de 2015. México.

Gobierno de México-Secretaría de Educación Pública (SEP). (2013). Modelo de Atención con Enfoque Integral para la Educación Inicial. En http://basica. sep.gob.mx/MODELO_ATENCION.pdf

Gobierno de México-Secretaría de Educación Pública (SEP). (2011). Programa de Estudio 2011. Guía para la Educadora. Educación Básica Preescolar. En http://www.reformapreescolar.sep.gob.mx/ACTUALIZACION/ PROGRAMA/Preescolar2011.pdf

Gobierno de México-Secretaría de Salud, Secretaría de Educación Pública, Secretaría de Relaciones Exteriores (2008). Declaración Ministerial: Prevenir con Educación. En http://www.censida.salud.gob.mx/descargas/ declaramin.pdf

Marina, M. (2010). La educación sexual integral en el jardín de infantes. En M. Marina (Coord.), Educación sexual integral para la educación inicial. Contenidos y propuestas para las salas (pp. 11-22). Argentina: Subsecretaría de Equidad y Calidad Educativa. Programa Nacional de Educación Sexual Integral. Dirección Nacional de Gestión Educativa. Dirección Nacional de Educación Inicial. Ministerio de Educación de la Nación.

Reybet, C. (2009). Construyendo un objeto de investigación desde la antropología que articule: género, escuela y primera infancia. Revista La aljaba, 13(13). En http://www.scielo.org.ar/scielo.php?script=sci_arttext\&p id $=$ S1669-57042009000100008

Rocha Sánchez, T. (2009). Desarrollo de la identidad de género desde una perspectiva psico-socio-cultural: un recorrido conceptual. Revista Interamericana de Psicología, 4(2), 250-259. En http://www.redalyc.org/articulo. oa?id=28412891006

Rosales, A. (2012). Introducción. En A. Rosales y S. Mino Gracia (Eds.). La salud sexual y reproductiva de jóvenes indigenas: Análisis y propuestas de intervención (pp. 11-17). México: Indesol.

Rosales, A. (2011). Sexualidad, derechos y violencia. Enfoques y conceptos para la enseñanza. México: Universidad Pedagógica Nacional. 
Rosales, A. (2010). Sexualidades, cuerpo y género en culturas indígenas y rurales. México: Universidad Pedagógica Nacional.

Salinas-Quiroz, F. (2014). Educación Inicial de Base Segura: Estudio observacional de los procesos internos de la educadora, de la interacción y del desarrollo sociocognitivo infantil. (Tesis inédita de doctorado). Ciudad de México, México: Universidad Nacional Autónoma de México.

Salinas-Quiroz, F. \& Posada, G. (2015). MBQS: Método de evaluación para intervenciones en apego dirigidas a primera infancia. Revista Latinoamericana de Ciencias, Sociales, Niñez y Juventud, 13(2). 1051-1063. En http:// revistalatinoamericanaumanizales.cinde.org.co/wp-content/ uploads/2015/08/MBQS_M\%C3\%A9todo_vol13n2a35.pdf

Salinas-Quiroz, F., Cambón V. \& Silva P. (2015). Aportes ecológico-interactivos a la psicología educativa. Revista Puertorriqueña de Psicología, 26(1), 26-27. En http://www.repsasppr.net/index.php/reps/article/viewFile/4/258

San Pelayo Ferrer, E. (2008). Educación de la sexualidad preescolar: Un enfoque alternativo y participativo, Revista SUMMA Psicológica, 5(1), 53-62. En https://dialnet.unirioja.es/descarga/articulo/2683148.pdf

Szasz, I. (1998). Primeros acercamientos al estudio de las dimensiones sociales y culturales de la sexualidad en México. En I. Szasz y S. Lerner (Eds.). Sexualidades en México (pp. 11-30). México: El Colegio de México.

Vygotsky, Lev S. (1991). Interacción entre enseñanza y desarrollo. En Selección de lectura de Psicología de las edades, 1. La Habana: Facultad de Psicología de la Universidad de la Habana.

Weeks, J. (1998). Sexualidad. México: Paidós-PUEG-UNAM. 